\title{
Análise de custos com pessoal e produtividade de equipes do programa de saúde da família em Fortaleza, Ceará
}

\author{
Costs with personnel and productivity analysis \\ of family health program teams in Fortaleza, Ceará
}

Fernando dos Santos Rocha Filho ${ }^{1}$

M arcelo Gurgel Carlos da Silva ${ }^{1}$

${ }^{1}$ Centro deCiências da Saúde, Universidade

Estadual do Ceará. Av. Paranjana, 1700, Itaperi. 60740-000 Fortaleza CE. fernandorocha1510@gmail.com
Abstract The research verified the productivity and the operational personnel costs of eight teams of the Family Health Program (PSF) of two Basic U nits of Family Health (UBASF) in Fortaleza, Ceará, through the methodology of costs by absortion, in 2004. The largest expenses were with personnel $(75 \%)$, mainly with the PSF teams, and medicines (18\%). The federal allocations received in September, 2004, by each PSF team, were $R \$ 9,543.33$. The total cost by team was $R \$ 15,719.00$. Some professionals of various PSF teams showed productivity above the set objectives, but the average productivity by doctor and nurse was below fifty per cent of objectives, with high idle time making the work onerous. The unity cost of home visits by doctor and prenatal attendance by nurses were checked, and, would be less expensive if the idle time were reduced. Various alternatives of cost reduction were observed, such as scale savings of some resources and services and re-negotiation of contracts with suppliers and cooperatives. The data obtained will contribute to more accurate planning for theinstallation and maintenance of PSF teams as well as alternatives of cost reductions, higher productivity, and better quality.

Key words Family Health Program, Health costs, Productivity, Health personnel, $\mathrm{H}$ ealth economic evaluation
Resumo Foram verificados a produtividade e os custos com pessoal das atividades de oito equipes de duas unidades do PSF de Fortaleza, Ceará, através da metodologia de custeio por absorção, em 2004. Os maiores gastos foram com pessoal (75\%), principalmente com os membros das equipes do PSF, e com medicamentos (18\%) ). Asverbas federais repassadas, em setembro de 2004, por equipe do PSF, foram de R \$ 9.543,33. 0 custo global da atuação da equipe foi de $\mathrm{R} \$ 15.719,00$. Alguns profi ssionais de várias equipes do PSF apresentaram produtividade acima das metas planejadas, porém a média geral das atividades por médico e enfermeiro nas equipes ficou abaixo da metadedaquelas metas, com alta capacidadeociosa e tornando as atividades muito onerosas. Verificou-se o custo unitário da visita domiciliar por médico eda consulta de pré natal por enfermeiro, que seriam menos onerosos se a capacidade ociosa fosse menor. $\mathrm{Ha}$ várias alternativas de minimização de custos, incluindo economia de escala relativa a alguns recursos e serviços e renegociação de contratos com empresas e cooperativas. As informações possibilitam o planejamento mais preciso, para manutenção e instalação de equipes, além de alternativas de menores custos com maior produtividade e qualidade. Palavras-chave Programa de Saúde da Família, Custos em saúde, Produtividade, Recursos humanos, Avaliação econômica em saúde 
Introdução

No Município de Fortaleza, Estado do Ceará, as primeiras equipes do PSF (Programa de Saúde da Família) começaram a atuar na década de $1990^{1}$. Atuavam em Fortaleza, em 1999, cinquenta ESF (Equipes de Saúde da Família)². Este número aumentou para 97 equipes no ano 2000, $101 \mathrm{em} 2001 \mathrm{e}$ manteve este número até pelo menos setembro de 2004 , cobrindo 348.450 pessoas ( $16 \%$ da população total de Fortaleza, pelos critérios do programa).

O PSF é mais do que um programa de saúde. Ele é uma estratégia de reformulação do sistema de atenção à saúde no Brasil, mormente no âmbito do Sistema Ú nico de Saúde (SUS) 2,3,4. O s objetivos do PSF se inserem na atenção básica de saúde (ABS), como primeiro nível do sistema local de saúde $e^{5,6}$. Seus recursos estão centrados nas equipes do PSF, que são compostas, atualmente, pelos seguintes profissionais de saúde?: um médico (preferencialmentegeneralista), um enfermeiro edequatro a dez agentes de saúde. Atualmente, estão sendo integrados cirurgiões-dentistas ao PSF, na proporção de um dentista para cada duas ESF.

Vem crescendo a necessidade de mais investigações eavaliações científicas queesclareçam o desempenho efetivo do PSF em cada realidade em que atua $8,9,10$. A lém das avaliações normativas dos serviços, ganham importância as pesquisas avaliativas ${ }^{11}$. AsESF têm sido efetivas quanto a mudanças positivas no estado de saúde das populações, ao contribuir, por exemplo, para a diminuição das internações hospitalares por vários agravos ${ }^{3,12,13}$.

0 custo da atenção à saúde é cada vez mais elevado, mesmo nos países mais ricos e, ainda assim, os recursos continuam sempre escassos frente ao crescente volume das necessidades em saú$\mathrm{de}^{14}$. Isso torna cada vez mais urgente o conhecimento sobre a formação dos custos em saúde, que subsidiem intervenções que melhorem a produção dos serviços a menores custos ${ }^{15-17}$. Essas novas alternativas também otimizam o desempenho das equipes, possibilitando melhores condições de trabal ho para os profissionais e os gestores, e meIhores ações de saúde para a comunidade. Por outro lado, a análise de custo não deve ser utilizada para medidas que reduzam a verba aplicada em saúde, em vista do "fosso" entre recursos e crescentes necessidades e demandas ${ }^{15}$.

O objetivo geral desta pesquisa foi identificar e analisar os custos com pessoal na produção das atividades de saúde das oito equipes do Programa de Saúde da Família (PSF) que atuavam em duas Unidades Básicas de Saúde da Família (UBASF) de Fortaleza, Ceará, em 2004. Para isto, foi necessário:
- Aferir o custo da atividade de saúde das oito equipes, identificando a composição dos recursos humanos empregados na sua produção;

- Avaliar a adequação do volume das ações de saúde produzidas, comparando esses volumes com o das metas do PSF de Fortaleza;

- Identificar as equipes que têm menores custos por ação de saúde, entre aquelas equipes que têm adequado volume de ações e adequada composição de recursos;

- Identificar alternativas para otimizar a atuação das equipes de cada unidade e de cada administração regional, minimizando seus custos, aperfeiçoando o seu desempenho, a utilização de recursos e a oferta de ações de saúde, contribuindo para as tomadas de decisão.

Os custos identificados nesta pesquisa são, principalmente, os custos gerados para a Prefeitura Municipal de Fortaleza (PM F), isto é, gastos realizados pela PM $F$ na atuação das equipes do PSF, como os gastos com recursos humanos. Os gastos desembolsados pelo Governo do Estado do Ceará e pelo Governo Federal são apresentados separadamente, sempre que ocorrem.

\section{Material emétodos}

0 universo desse estudo descritivo de natureza quantitativa são os custos, com pessoal, das ações de saúde de oito equipes do PSF de Fortaleza, sendo três equipes da UBASF José Valdevino de Carvalho, localizada no bairro da Itaoca, dentro da área subordinada à Secretaria de Administração Regional IV (SER IV), e cinco equipes da UBASF Terezinha Parente, que está localizada no bairro Curió, dentro da Regional VI (SER VI). 0 campo da pesquisa são as instalações de duas U BASF, cada uma apoiando equipes do PSF, e as sedes das duas respectivas Administrações Regionais.

Os dados coletados foram aqueles necessários para identificar os custos, com pessoal, das ações de saúde das equipes do PSF. Foram utilizadas a pesquisa em documentos e outros registros de recursos; aplicação de questionários com os gestores das UBASF e das duas Secretarias Regionais, e com os profissionais que executam as atividades; observação direta da área física, dos recursos, dos custos, das atividades, e outros aspectos. Foram feitos registros em formulários e em planilhas eletrônicas.

As etapas fundamentais das avaliações econômicas em saúde, inclusive das análises de custos, são apresentadas a segui ${ }^{16-20}$.

1. Descrever as estratégias de intervenção e alternativas a serem analisadas ( cada U BASF e cada 
equipe do PSF) edefinir os seus resultados esperados (ação global de saúde e ações de saúde específicas), os quais terão seus custos avaliados;

2. I dentificar e calcular todos os custos de cada intervenção, que são os gastos com os recursos utilizados na produção do empreendimento. Foi utilizado o método de custeio por absorção, em que os centros de custo finalísticos absorvem os custos dos centros de custos de apoio e de base, frequentemente utilizando ratei os para a alocação dos custos indiretos;

3. I dentificar e calcular o resultado (volume de ações de saúde) atingido por cada alternativa (cada ESF);

4. Confrontar os custos (de produção) e os resultados (ações de saúde) obtidos por cada intervenção, verificando sua eficiência;

5. Comparação das intervenções alternativas, em termos de custo-resultado. Identificar a(s) alternativa(s) mais eficiente(s);

6. Qualificar e revisar essa decisão à luz do risco, da incerteza e da sensibilidade.

Os indicadores de resultado de cada ação de saúde de cada equipe são os volumes mensais de ações produzidas/realizadas, observando se estão dentro dos limites mínimo e máximo aceitáveis definidos. As ações de saúde realizadas pelas equipes do PSF incluídas no estudo, são: 1. Atividade global da equipe do PSF (também denominada de atenção global à saúde da família) (recurso metodológico para a aferição do custo global da equipe do PSF); 2. Visita médica domiciliar; 3. Exame de pré-natal por enfermeiro. 0 critério de inclusão das ações de saúde no presente estudo foi 0 de terem um alto peso estimado dentro dos custos totais da produção da equipe e a relevância da atividade para a saúde pública.

As equipes que dão atenção à saúde das pessoas são consideradas áreas de produção finalística dentro do sistema de custos das UBASF estudadas. Utilizando-se o método de custeio por absorção ${ }^{21-24}$ para o cálculo do custo global da atuação decada equipe do PSF, define-se cada equipecomo um departamento produtivo ${ }^{23}$ dentro de cada UBASF e, também, como um centro de custos produtivo 23,24 .

0 mês de 2004, cujos custos e produção são analisados em cada unidade de saúde, foi escolhido observando-se o mês que mais refletiu o padrão do volume de produção de ações durante 0 ano.

As ações de saúde realizadas pelas equipes nas duas unidades de saúde pesquisadas são consideradas os serviços produzidos pela organização e prestados à clientela, sendo os produtos finais do processo produtivo. As equipes do PSF (EPSF) de cada UBASF, as equipes de odontologia e as do Programa de Saúde Escolar (PSE) são consideradas os departamentos produtivos das duas UBASF em termos de produção dos serviços finalísticos, pois el as produzem as ações de saúde para os clientes das UBASF.

0 conjunto das ações de saúde de cada equipe do PSF é aqui denominado de Atenção á Saúde da Família. São levadas em conta as ações de saúde que envolvem médicos e enfermeiros do PSF, com a participação dos outros membros das equipes. 0 número padrão de expedientes de quatro horas semanais previstos para cada atividade em cada equipe do PSF das duas UBASF é aquele apresentado na publicação da Escola de Saúde Pública do Ceará ${ }^{6}$, e corroborado pelas coordenadoras das duas UBASF.

Para a alocação dos gastos dos centros de custos de apoio, entre si e para as equipes do PSF, foram utilizados critérios de rateio bastante detaIhados ${ }^{21,25}$, como as horas dedicadas pelos profissionais, as áreas físicas utilizadas, número de atividades produzidas e outros. Os critérios são descritos a seguir:

- Serviço de transporte: número de expedientes/semana para visita domiciliar de cada equipe do PSF;

- Sala e equipamento para coleta para prevenção ginecológica: número de atendimentos na sala de ginecologia (profissionais: médico ginecologista e enfermeiro);

- SAM E: número de atendimentos por profissionais denível superior de cada centro de custos (CC) assistencial (profissionais: médico PSF, enfermeiro PSF, pediatra, odontólogo e ginecologista);

- Acolhimento e sinais vitais: número de atendimentos por profissionais de nível superior de cada CC assistencial (profissionais: médico PSF, enfermeiro PSF, pediatra, odontólogo e ginecologista);

- Esterilização: número de atendimentos por profissionais de nível superior de cada CC assistencial;

- Farmácia: número de atendimentos por profissionais de nível superior de cada CC assistencial (profissionais: médico PSF, enfermeiro PSF, pediatra, odontólogo e ginecologista).

- Serviços gerais ampliados: área física de cada CC;

- Almoxarifado: seu (pequeno) custo foi incorporado ao do setor de coordenação (este serviço éde pouco volumena UBASF, eér realizado pela coordenação);

- Coordenação da UBASF: número de horas trabalhadas em cada CC; 
- Gastos com telefonia: alocar ao CC coordenação (a coordenação centraliza os telefonemas na UBASF);

- Gastos com energia elétrica: objetos que consomem energia em cada CC;

- Gastos com água: área física de cada CC (o gasto maior é com a limpeza e higienização das áreas).

\section{Resultados ediscussão}

A Unidade Básica de Saúde da Família (UBASF) JoséValdevino de Carvalho (JVC) ea UBASF Terezinha Parente (TP) são organizações públicas da Prefeitura M unicipal de Fortaleza (PM F), prestam serviços de saúde do tipo ambulatorial edomiciliar ese incluem no nível da atenção básica de saúde do Sistema Ú nico de Saúde (SUS) de Fortaleza. Integradas à U BASF José Valdevino de Carvalho estavam três equipes do PSF denominadas de: equipe do PSF amarela, equipe do PSF azul e equipe do PSF vermelha. Integradas à UBASF Terezinha Parente estão cinco equipes do PSF denominadas de: equipe do PSF amarela, equipe do PSF azul, equipe do PSF lilás, equipe do PSF verde e equipe do PSF vermelha.

Equipes do PSF com mais adequados volumes de produção em setembro de 2004

Os custos com pessoal e a produtividade das equipes do PSF foram objeto de investigação no mês de setembro de 2004, que foi o último mês de 2004 em que se apresentaram típicas tanto a produção quanto a situação de recursos das equipes das duas unidades de saúde analisadas. 0 volume de produção médio mensal de visitas domiciliares, tanto por médico como por enfermeiro, detodas as equipes da U BASF JVC, manteve-se em nível estável de janeiro atéo fim de setembro de 2004. Sofreu queda importante em outubro, novembro e dezembro, sendo isto atribuído principalmenteà falta do veículo de transporte para as equipes nestes três meses. Os outros tipos de ações de saúde apresentaram queda menor nesse período.

$\mathrm{Na}$ UBASF TP não houve falta do serviço de transporte nos meses supracitados e nem queda do volume das visitas domiciliares. Nesta UBASF, o mês selecionado para análise inicial foi também o mês de setembro de 2004, para permitir comparações com a situação das equipes da U BASF JVC.

A equipe do PSF da UBASF JVC que apresentou, em setembro de 2004, mai or emais equilibrado volume de produção de ações de saúde, por médico e enfermeiro, foi a equipe amarela. Seu volume de produção do conjunto de ações por médico eenfermeiro atingiu o percentual de $36,22 \%$ em relação ao padrão definido nas normas do $\mathrm{PSF}^{6}$, enquanto a equipe vermelha atingiu $32,01 \%$ ea equipe azul, $28,20 \%$.

A EQPSF daUBASF Terezinha Parentequeapre sentou, em setembro de 2004, maior e mais equilibrado volume de produção de ações de saúde, por médico e por enfermeiro, foi a equipe vermelha, com desempenho global de $47,60 \%$ do planejado. A equipe verde do PSF da UBASF TP não contava com o médico em setembro de 2004 e não apresentou produção que envolvesse esse profissional.

As equipes amarela e vermel ha da UBASF JVC, e a equipe vermelha da UBASF TP foram as de melhor desempenho no mês de setembro de 2004, e as análises de custo- produtividade se concentrarão principalmente nestas três equipes, pelos motivos já levantados na discussão da metodologia. Em certos momentos, a análise da equipe vermeIha daU BASF JVC terá precedência sobrea da equipe amarela em vista de esta última ter custo atípico, bem menor que o das outras, pois a enfermeira da equipe tem vínculo com o M inistério da Saúde (MS), diminuindo o gasto desembolsado pela PM F.

\section{Organização dos centros de custos} das UBASF

As duas UBASF estudadas contam com outras equipes de saúde além das equipes do PSF, como as equipes deodontologia, de ginecologia eas equipes do Programa de Saúde Escolar (PSE), que incluem pediatras. Cada uma dessas equipes são consideradas departamentos e centros de custos deprodução finalística, independentes entresi. Estes centros de custos finalísticos, ou áreas finalísticas, são denominados de centros de custos assistenciais em outras classificações utilizadas ${ }^{21}$. Os centros de custos detectados nas duas unidades de saúde são apresentados na lista abaixo:

a) Centros de custos assistenciais (realizam as atividades finalísticas):

- Equipes do PSF (5 na UBASF Terezinha Parente);

- Equipe de odontologia;

- Equipe de ginecologia;

- Equipe do Programa de Saúde do Escolar (pediatria);

b) Centros de custos de apoio assistencial (fornecem bens eserviços apenas aos centros de custos assistenciais, listados acima):

- SAME (Serviço de Arquivo M édico);

- Acolhimento (faz a recepção, a aferição da 
temperatura, pressão arterial e peso; na UBASF Terezinha Parente, esta atividade é realizada pelos auxiliares de enfermagem das equipes do PSF);

- Esterilização (também função, na U BASF Terezinha Parente, dos auxiliares de enfermagem das equipes do PSF);

- Lavanderia (tem pouca importância nos custos das equipes do PSF na UBASF Terezinha Parente, pois a maioria dos tecidos utilizados são descartáveis);

- Farmácia.

c) Centros de custos de apoio geral (apóiam tanto os centros de custos assistenciais quanto os não assistenciais):

- Serviços gerais (limpeza, vigilância e manutenção de pequenos reparos);

- M anutenção de maiores reparos (serviço realizado fora da UBASF);

- Transporte (dá apoio às equipes do PSF e à coordenação da UBASF);
- Almoxarifado (cuja atividade é realizada, nas UBASF, pelo pessoal da coordenação).

d) Centro de custos administrativos e de gastos gerais:

- Coordenação da UBASF;

- Gastos com tel efonia, energia elétrica eágua.

Distribuição do pessoal

por centros de custos de cada UBASF

A Tabela 1 mostra a distribuição do pessoal por centros de custos na UBASF TP. Observa-se que o número de agentes comunitários de saúde por equipe é de quatro nas equipes lilás e vermeIha, e de três nas demais equipes, influenciando a variação de custos entre as equipes do PSF.

Os custos identificados nesta pesquisa são, principalmente, os custos para a Prefeitura Municipal de Fortaleza (PMF), isto é, gastos realizados pela PMF na atuação das equipes do PSF, como

Tabela 1. Distribuição do pessoal por Centros de Custos da UBASF TP em setembro de 2004.

\begin{tabular}{|c|c|c|c|c|c|c|c|c|c|c|c|c|c|c|}
\hline Função & Vínculo & 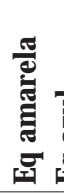 & & 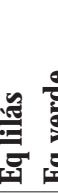 & 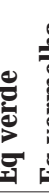 & 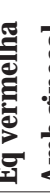 & $\begin{array}{l}\overline{8} \\
8 \\
0 \\
0 \\
0 \\
\text { है } \\
\end{array}$ & 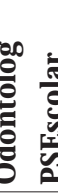 & 'ָ & 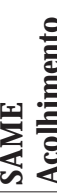 & 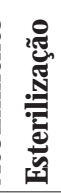 & 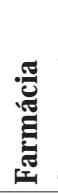 & 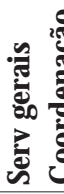 & 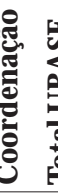 \\
\hline Agente C Saúde & EmprA & 0 & 2 & 0 & 1 & 3 & & & & & & & & \\
\hline Agente C Saúde & EmprB & 3 & 1 & 4 & 2 & 1 & & & & & & & & 1 \\
\hline Aux admnistr & Gov Ceará & & & & & & & & 1 & & & & & \\
\hline Aux admnistr & PM F Direta & & & & & & & & & & & & & 1 \\
\hline Aux admnistr e Aux da coord & PM F Direta & & & & & & & & & & & & & 1 \\
\hline Aux administr & EmprC & & & & & & & & & 2 & & & & \\
\hline Aux serv saúde & EmprC & & & & & & & 1 & & 2 & & 1 & & \\
\hline Aux serv gerais & PM F Direta & & & & & & & & & & & & & \\
\hline Aux enferm & MS & & & & & & & & & & & & & \\
\hline Aux enferm & EmprB & 1 & 1 & 1 & 1 & 0 & & & & & & & & \\
\hline Aux enferm & PM F Direta & & & & & 1 & & & & & & & & \\
\hline Coordenadora US & PM F Direto (Cargo direção) & & & & & & & & & & & & & 1 \\
\hline Dentista & Coop A & & & & & & & 1 & & & & & & \\
\hline Dentista & MS & & & & & & & 1 & & & & & & \\
\hline Dentista & SESA-CE & & & & & & & 1 & & & & & & \\
\hline Enfermeiro PSF & Coop A & 1 & 1 & 1 & 1 & 1 & & & & & & & & \\
\hline M édico PSF & Coop A & 1 & 1 & 1 & & 1 & & & & & & & & \\
\hline Pediatra PSE & PM F Direta & & & & & & & & 1 & & & & & \\
\hline Zelador & Empr E & & & & & & & & & & & & 3 & \\
\hline Total & & 6 & 6 & 7 & 6 & 7 & 0 & 4 & 2 & 4 & 00 & 1 & 3 & 34 \\
\hline
\end{tabular}

Obs: Equipeverdedo PSF da UBASF TP não contou com médico em setembro de 2004. 
gastos com recursos humanos, materiais e outros. Os gastos desembolsados pelo Governo do Estado do Ceará e pelo Governo Federal são apresentados separadamente, sempre que ocorrem.

$O$ gasto mensal global com cada equipe do PSF em termos de pessoal é, em sua maior parte, fixo, sendo constituído dos rendimentos e encargos pagos a cada profissional em atuação. Além disso, oitenta por cento de todos os profissionais que atuavam nas duas UBASF, incluindo aqueles que não faziam parte do PSF, eram vinculados a essas cooperativas e a empresas de serviços, em um processo chamado de "terceirização".

Verifica-sequeas três equipes do PSF naUBASF JVC têm o mesmo número de agentes comunitários de saúde, auxiliares de enfermagem, médicos e enfermeiros. A enfermeira da equipe amarela tem vínculo direto com a PM F e com o Ministério da Saúde, com gasto para a PM F bem menor que 0 gasto com os enfermeiros das outras equipes das duas UBASF. Esta é a principal causa do custo da equipeamarela da UBASF JVC ser significativamente menor que o custo das outras equipes do PSF.

Custos nas equipes do PSF

com pessoal próprio e com pessoal

dos centros de custos de apoio

A equipe do PSF que apresentou, ao mesmo tempo, alta produtividadee custo geral considerado típico, no mês desetembro de 2004, foi a equipe vermelha da UBASF Terezinha Parente (TP). Os gastos mensais gerais com os centros de custos de apoio na UBASF TP em setembro de 2004 estão apresentados na Tabela 2.

Os custos desses centros de custos contribuem em parte para os custos finais dos outros centros decustos, inclusive os de cada equipe do PSF, equipe de odontologia e outras. 0 centro de custos de apoio com maior custo básico (com pessoal) em setembro de 2004 foi o SAME, com R\$3.896,24. Seu custo, acrescentando 0 apoio da coordenação e de outros itens, foi de $R \$ 4.520,91$. 0 gasto com pessoal próprio da equipe verde da UBASF TP, isto é, com os membros da equipe, em setembro de 2004 , foi de $R \$ 4.891,21$, como pode ser observado nas Tabelas 3 e 4.0 gasto da equipe amarela da U BASF JVC foi de $R \$ 7.959,81$. Estes gastos diferiram muito dos gastos nas demais equipes, que variaram entre $R \$ 9.157,81$ e $R \$ 10.170,17$.

Estas informações podem auxiliar os gestores a encontrar dificuldades, ou vantagens, na situação de recursos das equipes com custos atípicos, os quais, no caso da equipe verde mencionada, deveram-se à ausência de médico na equipe, e, no caso da equipe amarela JVC, à presença, na equipe, de enfermeira cedida pelo M inistério da Saúde, importando em menores custos para a PM F.

As Tabelas 3 e 4 demonstram que a atuação da equipe vermel ha da U BASF Terezinha Parente teve custo com pessoal mensal global de $R \$ 11.856,48$. Este é o custo total com pessoal próprio da equipe e dos centros de custos que a apóiam. Observa-se, também, que, entre os seus gastos diretos e os dos departamentos e serviços que Ihe dão apoio, os gastos mais elevados foram os realizados com os

Tabela 2. Gastos mensais gerais com os Centros de Custos de Apoio na UBASF Terezinha Parente em setembro de 2004 $(\mathrm{R} \$ 1,00)$.

\begin{tabular}{|c|c|c|c|c|c|c|c|c|c|}
\hline Setor & Energia & Água & Telefone & Coordenação & Serv gerais & SAME & Farmácia & Acolhimento & Transportes \\
\hline Energia & $1.179,36$ & & & 119,37 & & 126,09 & 68,56 & 16,78 & \\
\hline Água & & 156,07 & & 14,48 & & 5,93 & 7,10 & 5,46 & \\
\hline Telefone & & & 532,94 & 532,94 & & & & & \\
\hline Coordenação & & & & $3.131,00$ & 289,68 & 386,44 & 96,61 & 48,30 & \\
\hline Serv. gerais & & & & 259,35 & $2.505,00$ & 106,22 & 127,15 & 97,85 & \\
\hline SAME & & & & & & $3.896,24$ & & & \\
\hline Farmácia & & & & & & & 871,91 & & \\
\hline Acolhimento & & & & & & & & & \\
\hline Transportes & & & & & & & & & $2.180,00$ \\
\hline Total & $1.179,36$ & 156,07 & 532,94 & $4.057,14$ & $2.794,68$ & $4.520,91$ & $1.171,33$ & 168,39 & $2.180,00$ \\
\hline
\end{tabular}

Obs: Não havia profissionais alocados exclusivamente no Acolhimento (esinais vitais) e esta função era realizada pelos auxiliares de enfermagem em rodízio. 
profissionais da equipe, $R \$ 9.780,10$ (82,49\%), seguidos daqueles dos seus centros de apoio, como o SAME, com R\$ 861,56 (7,27\%), a coordenação com $R \$ 556,70(4,70 \%)$ e o centro de serviços gerais com $R \$ 416,17$ (3,51\%). 0 acolhimento e a esterilização eram realizados, na UBASF TP, pelos auxiliares de enfermagem das equipes do PSF, e 0 gasto do apoio que estes setores receberam da coordenação e de serviços gerais foi muito pequeno, em relação aos outros gastos, sendo, por exemplo, $R \$ 27,20(0,23 \%)$, para o acolhimento. Esta é a principal razão do menor custo com pessoal de apoio na UBASF TP do que na UBASF JVC.

Os gastos com pessoal do SAME e serviços gerais são semel hantes nas duas U BASF. $O$ gasto com pessoal da coordenação na equipe vermelha da UBASF TP, de $\$ 556,70$ (4,70\%), foi maior do que na equipe vermelha da UBASF JVC, de R $\$ 247,94$ $(2,06 \%)$, em decorrência do maior número defuncionários (três) no setor de coordenação da primeira UBASF, enquanto que, na outra UBASF, só atua a coordenadora, havendo a carência de pelo menos um auxiliar de apoio.

0 gasto total com o pessoal dos centros de apoio à equipe vermelha do PSF da U BASF Terezinha Parenteficou em 17,51\% (R\$2.076,37), o qual, somado ao gasto de $R \$ 9.780,00(82,49 \%)$ com pessoal próprio da equipe do PSF, perfaz o custo total de $\mathrm{R} \$ 11.856,48 \mathrm{com}$ pessoal. Este gasto de $17,51 \%$ com pessoal dos centros de apoio à equipe vermel ha do PSF da UBASF TP deFortaleza reflete uma estrutura deapoio mais robusta quenas equipes de Crateús, Cearáa ${ }^{6}$.

Tabela 3. Gastos com pessoal próprio das equipes do PSF e com pessoal de centros de apoio na UBASF Terezinha Parente em setembro de $2004(R \$ 1,00)$.

\begin{tabular}{|c|c|c|c|c|c|c|c|c|c|}
\hline Setores & Eq amarela & $\%$ & Eq azul & $\%$ & Eq lilás & $\%$ & Eq verde & Eq vermelha & $\%$ \\
\hline Pess Coor & & & & & & 4, & & & 4,7 \\
\hline Pess Serv & & & & & & & & & 3,5 \\
\hline Pess SAME & & 6, & & 7, & & 6,91 & & & 7,27 \\
\hline Pess Farmáci & & & & & & 1,72 & & & 1,8 \\
\hline to & 51 & 0 & & 0 , & 57 & 0,22 & 19 & 20 & 0,23 \\
\hline Total pess apoio & $1.771,17$ & 15,81 & $1.878,22$ & 17,02 & $2.006,86$ & 16,48 & $1.149,24$ & 076,37 & 17,51 \\
\hline Profission. Equipe & $9.433,69$ & 84,19 & & 82, & $10.170,17$ & 83,52 & & 10 & 82,4 \\
\hline Total pessoal & $11.204,86$ & 100,00 & $11.036,02$ & 100,00 & $12.177,03$ & 100,00 & $6.040,43$ & $11.856,48$ & 100,00 \\
\hline
\end{tabular}

Obs: Equipeverde do PSF daUBASF TP não contou com médico em setembro de 2004.

Tabela 4. Comparativo dos gastos com pessoal na equipe vermelha da UBASF JVC e na equipe vermelha da UBASF TP em setembro de 2004 (R\$ 1,00).

\begin{tabular}{lrrrrrr}
\hline \multicolumn{1}{c}{ Setores } & Eq amarela JVC & \multicolumn{1}{c}{$\%$} & Eq vermelha JVC & \multicolumn{1}{c}{$\%$} & Eq vermelha TP & $\%$ \\
\hline Pessoal da Coordenação & 247,94 & 2,31 & 247,94 & 2,06 & 556,7 & 4,70 \\
Pess. Serv gerais & 552,58 & 5,15 & 574,17 & 4,78 & 416,17 & 3,51 \\
Pess. SAM E & 854,95 & 7,97 & 824,2 & 6,86 & 861,56 & 7,27 \\
Pess. Acolhimento & 611,83 & 5,70 & 589,83 & 4,91 & 27,2 & 0,23 \\
Pess. Esterilização & 95,46 & 0,89 & 92,03 & 0,77 & - & 0,00 \\
Pess. Farmácia & 409,36 & 3,81 & 394,64 & 3,28 & 214,74 & 1,81 \\
Subtot 1-6 (Pess CC Apoio) & 2772,12 & 25,83 & 2722,81 & 22,66 & 2076,37 & 17,51 \\
Pessoal Equipe PSF & $7.959,81$ & 74,17 & $9.292,75$ & 77,34 & $9.780,10$ & 82,49 \\
Total (Pessoal 7-8) & $10.731,93$ & 100,00 & $12.015,56$ & 100,00 & $11.856,47$ & 100,00 \\
\hline
\end{tabular}

Obs: Equipeverdedo PSF da UBASF TP não contou com médico em setembro de 2004. 
Importância do custo com pessoal nos custos globais das equipes do PSF

Entre os gastos com cada tipo de recurso empregado na equipe vermel ha da UBASF TP, os custos mais elevados foram, primeiramente, aqueles devidos ao pessoal próprio da equipe, $R \$ 9.780,10$ $(62,22 \%)$, depois a medicamentos, $R \$ 2.966,61$ $(18,87 \%)$ e, em terceiro, ao pessoal dos departamentos de apoio à equipe, $R \$ 2.076,37(13,21 \%)$. Com 0 acréscimo de transporte $(R \$ 554,00)$ e de energia, água e tel efonia ( $R \$ 351,49$ ), o gasto total com a equipe vermelha TP foi de $R \$ 15.719,57$. Os gastos com os profissionais da equipe do PSF somados àqueles com pessoal dos departamentos de apoio resultam em um gasto total na equipe, com pessoal, de $\mathrm{R} \$ 11.856,47$, correspondendo a $75,42 \%$ do custo global da equipe.

Verifica-se, portanto, a grande preponderância dos gastos com pessoal no custo da atuação global da equipe, o que coincidecom os resultados outros estudos sobrecustos de equipes do PSF $9,10,26$.

\section{Situações de minimização de custos}

Os achados do estudo ora apresentado podem contribuir para gerenciar melhor os custos, propiciando alternativas que reduzam os custos da atuação das equipes do PSF, sem diminuição da produtividade e da qualidade. Os valores das margens de lucro das empresas de serviços e das taxas de administração das cooperativas descritas mais acima, bem como o conhecimento dos vários valores pagos aos profissionais, podem auxiliar nos estudos da situação dos contratos.

Os custos podem ser diminuídos, também, em relação ao pessoal de apoio, como ocorreu na UBASF Terezinha Parente, ondehá cinco equipes, e os setores de esterilização eacolhimento, por exemplo, podem ser cobertos pelos próprios auxiliares de enfermagem das equipes do PSF, pois são cinco, gerando economia de escala.

\section{Desempenho de custo-produtividade}

O bservou-se que os custos das atividades conjuntas por médico e enfermeiro da maioria das equipes foi de $R \$ 6.398,00$ mensais, com exceção da equipe amarela JVC e verde TP; 0 fator mais determinante da variação do custo-produtividade, entre as equipes, foi a produtividade de cada equipe. A média alcançada, para o conjunto de atividades, em setembro de 2004, pelo médico e enfermeiro da equipe amarela da UBASF JVC, foi de $36,22 \%$ da produção esperada pelas metas do PSF local, sendo o melhor desempenho nesta UBASF. A equipe vermelha da UBASF TP atingiu 47,70\% das metas esperadas, sendo a de melhor custoprodutividade entre todas as oito equipes. A equipevermelha JVC atingiu 32,01\% das metas esperadas, ea equipeazul, TP 41,92\%. As demais equipes tiveram produtividade abaixo de $30 \%$.

Percebe-se que a produção média mensal de ações de saúde por parte de médicos e enfermeiros de todas as oito equipes estudadas está abaixo da metade do volume esperado; sendo, assim, a capacidade ociosa foi de mais de $50 \%$. Por outro lado, houve, no mês, alguns dias em que a capacidade ociosa com os mesmos profissionais, utilizando os mesmos equipamentos, salas e recursos, foi bem menor que $50 \%$.

$\mathrm{H}$ avia duas equipes na UBASF Terezinha Parente que programavam apenas um expediente de quatro horas semanais para visitas domiciliares por médicos e enfermei ros. Um médico do PSF, no entanto, chegou a realizar 94 visitas em um mês, correspondendo a onze visitas por expediente. $\mathrm{N}$ enhuma das oito equipes programou formalmente horários para a educação em saúde por enfermeiro e só muito raramente esta atividade era realizada. A análise de custo-produtividade das equipes demonstrou que o volume de todos os tipos de ações produzidas pode ser significativamente aumentado na situação já existente de pessoal e de outros recursos, sem necessariamenteincorrer em gastos adicionais, com exceção das visitas domiciliares, pois necessitam da reativação do serviço de transporte.

\section{Situação dos recursos}

0 quadro de profissionais não se alterou, em 2004, na maioria das equipes; porém, em alguns meses, duas equipes da UBASF TP não contaram com médicos. $O$ número de agentes comunitários de saúde era de quatro em duas equipes da UBASF $T P$, mas era de três nas restantes seis equipes analisadas, ficando, portanto, abaixo do recomendado pelas diretrizes do PSF, que é de, no mínimo, quatro ou cinco agentes por equipe. É importante ressaltar, ainda neste aspecto da situação de recursos, que, a partir de outubro de 2004, faltou o serviço de transporte na UBASF JVC.

Em relação à situação dos recursos, uma das dificuldades mais citadas foi a ausência de médico na equipe, o que compromete bastante tanto 0 desempenho quanto a captação de receitas, pois "o PAB variável do PSF pago como incentivo do $\mathrm{Mi}$ nistério da Saúde só é viabilizado com a presença desse profissional na equipe"26. Das oito equipes 
deFortaleza ora analisadas, duas equipes daU BASF TP estavam sem médico em vários meses do ano de 2004.

A chamada terceirização de vários serviços de apoio às atividades de equipes do PSF, encontrada nesta pesquisa e em outras ${ }^{26}$, é forma "precária" de contratação ${ }^{27}$ e era, em 2001, mais praticada nos municípios da Região Norte( $87 \%$ dos municípios), e Nordeste $(85,4 \%)$, que na Região Sul $(62,5 \%)^{27}$.

Custos com pessoal e receitas

nas equipes do PSF

0 total estimado do repasse mensal de verbas federais por equipe do PSF foi de R \$ 9.543,33 (Tabela 5). Como o gasto com a principal equipe analisada (equipe vermelha da UBASF Terezinha Parente) foi de $R \$ 15.719,00$, incluindo medicamentos, o repasse federal de $R \$ 9.543,33$ cobre $60 \%$ do gasto e a diferença a ser coberta pela PMF ( $R$ \$ $6.175,67)$ é de $39,20 \%$. 0 repasse federal cobre $97,57 \%$ do gasto com os profissionais da equipe do PSF (R $\$ 9.780,10)$ e cobre $80,49 \%$ do gasto com estes profissionais somado ao do pessoal dos centros de custos de apoio ( $R \$ 11.856,47)$.

\section{Conclusão}

Ao serem verificados eanalisados, em 2004, os custos com pessoal e a produtividade de oito equipes do PSF de duas U BASF de Fortaleza, utilizando-se a metodologia de custeio por absorção, foram detectadas várias alternativas para otimização do desempenho dessas equipes. Possibilitou-se, assim, maior precisão na avaliação e no planejamento, do curto ao longo prazo, sobre a manutenção e atuação das equipes existentes e daquelas a serem instaladas.

Foi analisada a estrutura de custos da atuação das equipes, observando-se o tipo de recurso envolvido, o custo por cada departamento e centros de custo, a relação custo-produtividade das equipes do PSF e o financiamento dessas equipes. As informações verificadas pelo presente estudo preenchem uma lacuna importante nas análises existentes sobre o PSF em Fortal eza e possibilitam planejar alternativas para a otimização dos serviços, com melhoria da produtividadeeda qualidade, sem aumento de gastos, bem como alternativas para diminuição decustos, sem perda de produtividade.

\section{Colaboradores}

FS Rocha Folho trabalhou na concepção teórica, metodologia, trabal ho de campo, coleta e análise de dados e redação. M GC Silva também trabaIhou na concepção teórica, além de parte da metodologia, redação, análise e revisão.

Tabela 5. Repasses estimados de verbas federais por equipe do PSF de Fortaleza em setembro de $2004(R \$ 1,00)$.

\begin{tabular}{lrrrrr}
\hline \multicolumn{1}{c}{ Receitas } & \multicolumn{1}{c}{ Ao ano } & \multicolumn{1}{c}{ Ao mês } & No agentes CS & Pop coberta/equipe & Total/mês \\
\hline PSF/equipe & $33.360,00$ & $2.780,00$ & 4,00 & $2.780,00$ \\
PACS/Agente CS & & 260,00 & & $1.040,00$ \\
PAB fixo/hab & 13,00 & 1,08 & $4.000,00$ & $4.333,33$ \\
Vigil à saúde/ hab & 2,42 & 0,20 & $4.000,00$ & 806,67 \\
Visa Básica/hab & 0,25 & 0,02 & $4.000,00$ & 83,33 \\
Assist Farmacêutica Básica & 1,50 & 0,13 & $4.000,00$ & 500,00 \\
Total & & & $9.543,33$ \\
\hline
\end{tabular}

Fonte: Secretaria M unicipal deSaúdedeFortaleza. 


\section{Referências}

1. Fonseca MIF. Programa Saúde da Família de BeberibeCE [dissertação]. Fortaleza (CE): Universidade Federal do Ceará; 1997.

2. Sousa M F, organizadora. Os sinais vermelhos do PSF. São Paulo: Hucitec; 2002.

3. Paim JS. Saúde, política e reforma sanitária. Salvador: Instituto de Saúde Coletiva; 2002.

4. Andrade LOA, Barreto ICHC, Fonseca CD, Harzheim E. A Estratégia Saúde da Família. In: Duncan BB, Schmidt MI, Giugliani ERJ, organizadores. M edicina ambulatorial: condutas de atenção primária baseadas em evidências. Porto Alegre: Artmed; 2004. p. 88-100.

5. M arques RM, M endes A. A dimensão do financiamento da atenção básica e do PSF no contexto da Saúde - SUS. In: Sousa, M F, organizadora. Os sinais vermelhos do PSF. São Paulo: Hucitec; 2002. p. 71-101.

6. Ceará. Secretaria de Saúde do Estado. Escola de Saúde Pública do Ceará. Curso introdutório em saúde da família. Fortaleza: Escola de Saúde Publica do Ceará; 2002.

7. Brasil. Ministério da Saúde. Saúde da família: uma estratégia para a reordenação do modelo assistencial. 2a ed. Brasília: M inistério da Saúde; 1998.

8. M edina M G, Aquino R. Avaliando o Programa de Saúde da Família. In: Sousa M F, organizadora. Os sinais vermelhos do PSF. São Paulo: Hucitec; 2002. p. 135-151.

9. Darede CO, Santana EM, Lôbo JAL. Avaliação Econômica do PSF em municípios do Estado da Bahia. In: Anais do VI Encontro Nacional de Economia da Saúde; 2002; Nova Friburgo.

10. Pinto SD, Marinho M, Drachler M L, Cortes SM V Castro JD. Análise da evolução dos custos do Programa de Saúde da Família de Porto Alegre 1998 - 2002. In: Anais do VII Congresso Brasileiro de Saúde Coletiva; 2003; Brasília.

11. Contandriopoulos AP, Champanhe F, Denis JL, Pineault R. A avaliação na área de saúde: conceitos $e$ métodos. In: Hartz ZM A. Avaliação em Saúde. Rio de Janeiro: Fiocruz; 1997.

12. M iranda AS. Reorganização da rede pública dos serviços de saúde em Quixadá, CE [dissertação]. Fortaleza (CE): Universidade Estadual do Ceará; 1999.

13. Rocha Filho FS. Evolução do perfil das internações hospitalares após a implantação do PSF - Programa de Saúde da Família em Beberibe - Ceará. In: Anais do XXXIII Congresso Anual do Centro M édico Cearense / XII Outubro M édico; 1996; Fortaleza.

14. Silva MGC. Economia da saúde: da epidemiologia à tomada de decisão. In: Rouquayrol MZ, Almeida Filho N. Epidemiologia \& saúde. $6 \underline{\underline{a}}$ ed. Rio de Janeiro: M edsi; 2003.
15. Organización Mundial de la Salud. Economia aplicada a la sanidad. Ginebra: OM S; 1976.

16. Drummond M F, O'Brien BJ, Studart GL, Torrance GW. $M$ ethods for the economic evaluation of health care programmes. $2^{\text {nd }}$ ed. New York: Oxford University Press; 1997.

17. Witter S, Ensor TM, Jowett M, Thompson R. Health economics for developing countries: a practical guide London: M acmillan; 2000.

18. Ugá MAD. Instrumentos de avaliação econômica dos serviços de saúde: alcances e limitações. In: Piola SF, Vianna SM . Economia da saúde. 3a ed. Brasília: IPEA; 2002.

19. Aguilar MJ, Ander-Egg E. Avaliação de serviços e programas sociais. 2ª ed. Petrópolis: Vozes; 1995.

20. Knapp M. La economia de los servicios sociales. BarceIona: Euge; 1989.

21. Ching HY. Manual de custos de instituições de saúde. São Paulo: Atlas, 2001.

22. Bittencourt ONS. Sistemas tradicionais de custos Exemplo de uma instituição de saúde: Hospital Universitário de Porto Alegre. In: Ching HY. Manual de custos de instituições de saúde. São Paulo: Atlas; 2001.

23. Martins E. Contabilidade de custos. 8a ed. São Paulo: Atlas; 2001.

24. Medici AC, M arques RM . Sistema de custos como instrumento de eficiência e qualidade dos serviços de saúde. Cadernos FUNDAP (Qualidade em Saúde) 1996; 19(1):47-59.

25. Rocha Filho FS. Análise de custos e produtividade de equipes do Programa de Saúde da Família em Fortaleza - Ceará em 2004 [dissertação]. Fortaleza (CE): Universidade Estadual do Ceará; 2005.

26. Sousa MHL. Análise dos custos do Programa Saúde da Família: 0 caso de Crateús (CE) [dissertação]. Fortaleza (CE): Universidade Estadual do Ceará; 2000.

27. Girardi SN, Carvalho CL. Contratação e qualidade do emprego no Programa de Saúde da Família do Brasil. In: M inistério da Saúde/Falcão A, Santos N eto PM, Costa PS, Belisário SA, organizadores. Observatório de recursos humanos em saúde no Brasil. Rio de Janeiro: Fiocruz; 2003.

Artigo apresentado em 29/05/2006

Aprovado em 12/12/2006

Versão final apresentada em 01/02/2007 